\title{
Near Infrared Single Photon detection using an Avalanche photodiode Operated with a Bipolar gating signal
}

\author{
by B.K. Park, M.K. Woo, S.W. Han, Sung Moon
}

\author{
Center for Quantum Information, Korea Institute of Science and Technology, Seoul 136-791, Korea, \\ s.moon@kist.re.kr
}

\begin{abstract}
Near infrared single photon detection using an InGaAs/InP avalanche photodiode(APD) operated with a bipolar rectangular gating signal was reported. The use of the bipolar gating pulses enabled us to operate the APD well below the breakdown voltage during the gate-off time. As a result, it permits to decrease the lifetime of the trapped carriers, and then reduces the after-pulse noise of the detector. At a repetition rate of $200 \mathrm{MHz}$, the after-pulse probability is $8.2 \%$ less than conventional gating signal detection.
\end{abstract}

\section{Introduction}

The detection of single photons has attracted the attention of scientists since many years. In quantum communication, especially the quantum key distribution(QKD) based on optical fiber links, the telecommunication wavelength of $1.55 \mu \mathrm{m}$ is the most desirable for long-distance transmission, since optical fibers exhibit low dispersion and minimum loss $(0.2 \mathrm{~dB} / \mathrm{km})$ at this wavelength. Commercially available InGaAs/InP single photon avalanche diodes(SPADs) present single photon detection efficiencies up to $30 \%$ at telecom wavelengths. ${ }^{1)}$ To achieve single photon resolution in the detection process, InGaAs/InP APDs are usually operated above the breakdown voltage ( $\left.V_{B R}\right)$. This operation mode is called Geiger mode. In Geiger mode, an incoming photon is absorbed and creates an electronhole pair. The charge carriers grow into a self-sustaining current pulse due to the process of impact ionization. This multiplication phenomenon is known as an avalanche. During an avalanche event, some charge carriers are trapped by defects in the SPAD's multiplication layer. These carriers can be subsequently released and create undesired avalanches, called after-pulses. Generally, the after-pulsing effect is one of the major bottlenecks limiting InGaAs/InP SPADs performances for high frequency operation due to the need to apply long dead times. ${ }^{2-3)}$ There has been much effort to invent after-pulse suppression schemes, ${ }^{4-10)}$ in order to make high-speed SPDs and apply them to quantum information technologies, especially to QKD systems. In this paper, we present a simple and easy to implement method for reducing the after-pulse noise in InGaAs/InP APDs based SPDs that utilizes a bipolar gating signal instead of square gating pulse. To evaluate the effect of the bipolar gating signal, we compared its after-pulse probability per gate pulse with that of conventional square gating one. The results clearly indicate the effectiveness of the demonstrated detection method for reducing the after-pulse generation in InGaAs/InP SPADs.

\section{Experimental Methods}

The schematic diagram of the experimental setup used to characterize the performance of InGaAs/InP APDs operated with a bipolar rectangular gating signal is depicted in Fig. 1. The signal generator produces a periodic series of bipolar rectangular voltage pulses. These pulses are superimposed upon an offset voltage from a DC power supply to operate the APD in gated Geiger mode. At the same time, the signal generator triggers a short pulse generator that provides a trigger signals for a gain-switched distributed feedback laser diode (DFB-LD; NEC NX 8563LB). The laser diode generates short coherent pulses at $1.55 \mu \mathrm{m}$. These pulses were attenuated to the single photon level $(0.1$ photons/pulse) by an optical attenuator; it is then sent for detection to a pigtailed InGaAs/InP APD (EG\&G C30645). The time delay was set so that the photons impinging on the APD's sensitive area were synchronized with the gate-on state. The avalanche signals are picked up by measuring the voltage drop across the series resistor R1. These signals from the APD were then amplified using a high-speed amplifier (Analog Devices AD8353-EVALZ), and then registered by a counter (Credix FC-300). To obtain an accurate value of the count with reasonably small uncertainty, we connected the photon counter to a laptop computer (LabVIEW Interface) using a GPIB controller for hi-speed USB (National Instruments), and then we accumulated the pulse counts during long times to get the average values.

\section{Results and Discussions}

In gated Geiger mode operation, the time delay between the gate pulses and the incident photons must be well synchronized in order to ensure the highest quantum efficiency of the detector. The digital delay generator was used as the system clock for the experiment. The synchronization was made by varying the delay of the digital delay generator to maximize the counts rate. Photons are detected only when they arrive within the gate duration defined by the applied 
gate pulses. Photons arriving between gate pulses are not detected, evidenced by the fact that the total counts rate fall to the dark counts rate level. The full width at half maximum (FWHM) of the photon detection peak is around 900 ps. The data was measured at a fixed temperature of $223 \mathrm{~K}$, a quantum efficiency of $15 \%$, a gate repetition rate of $10 \mathrm{kHz}$, and a mean photon number per gate pulse of 0.1 photons/pulse. To evaluate the effect of the bipolar gating signal on the afterpulse noise of the detector, we compared its after-pulse probability per gate pulse using a bipolar gating signal with that of conventional SPD using a square gating pulse.

The breakdown voltage of the APD is - $56 \mathrm{~V}$ at the operating temperature of $223 \mathrm{~K}$. The bias voltage was set at $0.5 \mathrm{~V}$ below the breakdown voltage. These operating conditions yield a quantum efficiency of $15 \%$ and a dark count probability per gate pulse of $4 \times 10^{-5}$. To measure the after-pulse probability of the detector, we applied two successive gate pulses with the same height and width of $5.8 \mathrm{~V}$ and $2 \mathrm{~ns}$, respectively. To ensure the occurrence of an avalanche, we set the light intensity at the first gate pulses, and then no photon was introduced at the following second gate pulses. The afterpulse probability per gate pulse is calculated from the counted number of avalanches coming from the second gates after subtracting the dark counts measured under the same conditions.

Figure 2 shows a comparison of the after-pulse probability per gate pulse measured at different time intervals for an APD operated with conventional gating pulse (solid squares) and the demonstrated detection method using the bipolar gating signal (solid circles). It appears clearly from Fig. 2 that using a bipolar gating signal instead of a square gating pulse, which means operating the APD well below the breakdown voltage during a short period of the gate-off time (equals to the gate-on time), significantly reduces the after-pulse probability of the detector. This phenomenon can be explained by the fact that this operation mode decreases the electric field intensity across the APD during the gate-off time, and then reduces the lifetime of the trapped charge carriers, ${ }^{7)}$ which causes a reduction of the after-pulse probability of the detector. From Fig. 2, we can see that when the time interval between successive gate pulses is 5 ns, which corresponds to a repetition rate of the gate signal of $200 \mathrm{MHz}$ in the single gate mode, the after-pulse probability with bipolar gating signal is $8.2 \%$ less than that of conventional SPD operated with square gating pulse. The dark count probability is same for both methods.

\section{Conclusions}

In this paper, we have demonstrated a NIR single photon detection using an InGaAs/InP APD operated with a bipolar rectangular gating signal. The results obtained here clearly indicate the effectiveness of the demonstrated detection

method for reducing the after-pulse generation in SPADs. By applying our SPD to a quantum cryptography system, the quantum bit error rate (QBER) would be significantly decreased.

\section{Figures}

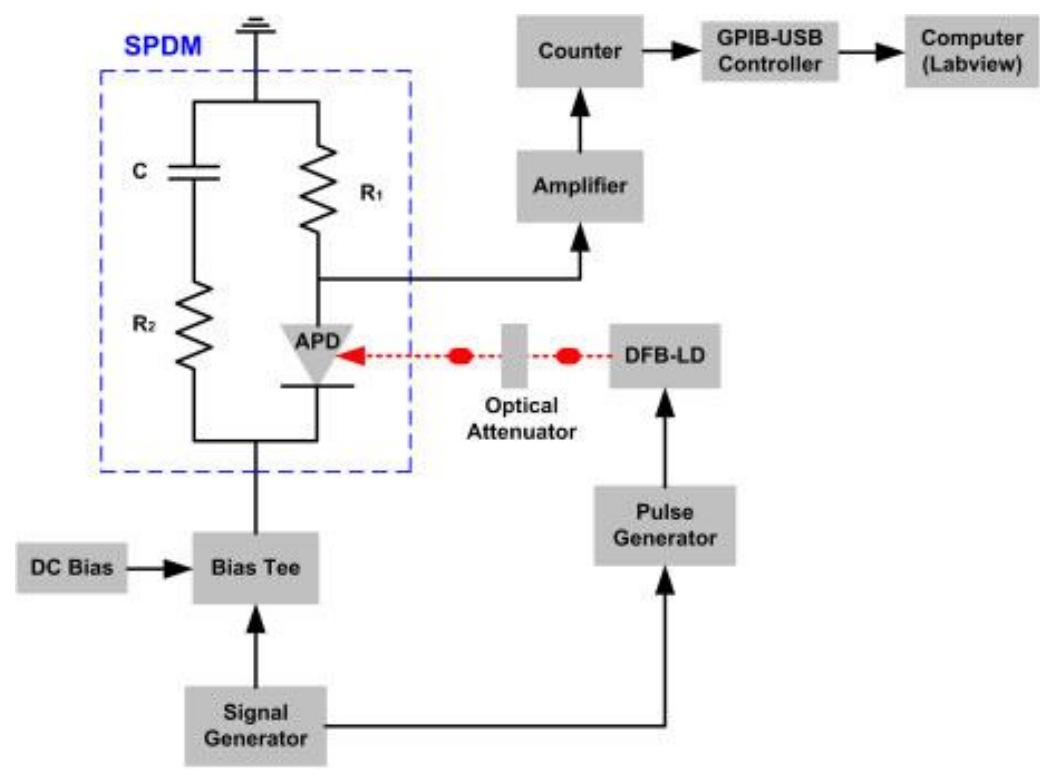

Fig. 1. Experimental setup used to characterize the performance of a SPD operated with a bipolar rectangular gating signal. Black lines and red dots indicate the electrical and optical paths, respectively.

The network in the blue dotted box is the single photon detector module (SPDM). 


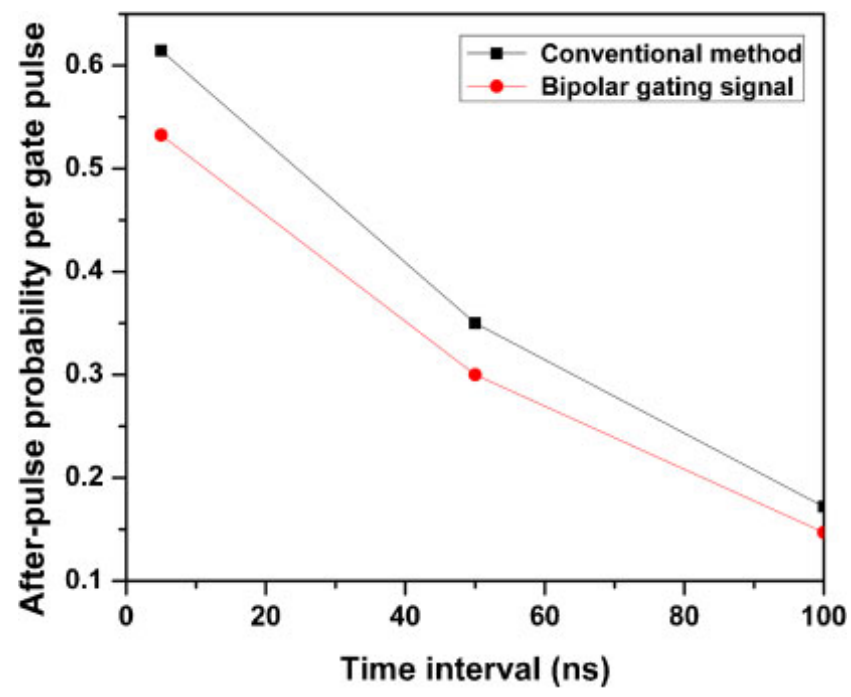

Fig. 2. Comparison of the after-pulse probability per gate pulse as a function of the time interval for APD with conventional gating pulse (solid squares) and bipolar rectangular gating signal (solid circles).

\section{REFERENCES}

[1] L. Xu, E. Wu, X. Gu, Y. Jian, G. Wu, and H. Zeng: Appl. Phys. Lett. 94 (2009) 161106.

[2] A. Yoshizawa, R. Kaji, and H. Tsuchida: Jpn. J. Appl. Phys. 41 (2002) 6016.

[3] D. S. Bethune, W. P. Risk, and G. W. Pabst: J. Mod. Opt. 51 (2004) 1359.

[4] N. Namekata, S. Sasamori, and S. Inoue: Opt. Express 14 (2006) 10043.

[5] Z. L. Yuan, B. E. Kardynal, A. W. Sharpe, and A. J. Shields: Appl. Phys. Lett. 91 (2007) 041114.

[6] N. Namekata, S. Adachi, and S. Inoue: Opt. Express 17 (2009) 6275.

[7] G. Ribordy, N. Gisin, O. Guinnard, D. Stuck, M. Wegmuller, and H. Zbinden: J. Mod. Opt. 51 (2004) 1381.

[8] A. Bouzid, J. B. Park, and S. Moon: J. Korean Phys. Soc. 56 (2010) 1418.

[9] X. Chen, E. Wu, G. Wu, and H. Zeng: Opt. Express 18 (2010) 7010.

[10] J. Zhang, R. Thew, C. Barreiro, and H. Zbinden: Appl. Phys. Lett. 95 (2009) 091103. 